\title{
Impact of Psycho-Social Factors on Non-Adherence: A Case Study of Chronic Kidney Disease (CKD)
}

\author{
Ghulam Ishaq, Fatima Kamran, Rafia Rafique, Shiza Sheerazi
}

\begin{abstract}
Chronic Kidney Disease (CKD) is progressively increasing in South Asian countries like Pakistan. This case study is describing, the psychosocial factors of non-adherence (medication, fluid and dietary). The patient was 19 years un-married male, diagnosed with End Stage Renal Disease (ESRD) and was on Hemodialysis for 3 years i.e. 2013 on thrice a week basis. He was rickshaw driver and first born among 2 siblings; 1 sister and 1 brother. He was referred to psychological assessment for non-adherence (medication, dietary and fluid). He had developed Drug Induced i.e. NSAID (Non-Steroidal AntiInflammatory Drugs) ESRD at the age of 16 years. He had secondary Hypertension and was presented with the physical complaints of fatigue, shortening of breath due to low $\mathrm{Hb}$ level. Psychosocial assessment was done by taking detailed case history interview assessing illness perceptions, health locus of control, health beliefs, perceived health status, doctor-patient relationship, adherence (medication, dietary and fluid) and life orientation. Target areas identified as result of psychosocial assessment, included non-adherence (medication, fluid and dietary), dissatisfactory doctor patient relationship, poor health status, pessimistic life orientation, mal-adaptive health beliefs and low socioeconomic status. 12 sessions have been conducted with the patients. He was psycho-educated relevant to his financial management plan due to distress of low socio-economic status. Insight in patient about the non-adherence (medication, dietary and fluid) was developed through CBT specific technique i.e. cost and benefits analysis. Counseling was given to him by maximizing the positive aspect of his life for his pessimistic life orientation. He was psycho-educated for his dissatisfactory doctor patient relationship. Coping statements was given to him to improve his self-efficacy relevant to his condition.
\end{abstract}

KEYWORDS. Chronic kidney disease, psychosocial factors, non-adherence, doctor patient's relationship.

This article may be cited as: Ishaq G, Kamran F, Rafique R, Sheerazi S. Impact of Psycho-Social Factors on Non-Adherence: A Case Study of Chronic Kidney Disease (CKD). J Liaquat Uni Med Health Sci. 2020;19(02):142-44. doi: 10.22442/jlumhs.201920678

\section{INTRODUCTION}

Non-adherence has also been noted to be particularly problematic in individuals with Chronic Kidney Disease $(C K D)^{1}$. The previous studies findings showed that patients' beliefs about their disease and its treatment have been proposed to be central to adherence. Specifically, the adaptive health belief was predictor of adherence and maladaptive health beliefs was predictor of non-adherence ${ }^{2}$.

Pervious literature demonstrated that demographic like socioeconomic factors and age (younger) leads the patient towards non-adherence of doctor advice ${ }^{3}$. As, for instance, studies using pharmacy refill data have reliably demonstrated the impact of higher medication price on non-adherence ${ }^{4}$.

\section{CASE REPORT}

The patient was 19 years un-married male, diagnosed with ESRD three years back i.e. 2013 at the age of 16 years and was on hemodialysis. He used selfmedication (Drug Induced) i.e. NSAID due to which he diagnosed with drug induced ESRD.

The patient's father was at the age 60 years and his mother was at the age of 55 years. His father was not working yet due to his old age. But 5 years in back he worked as a farmer. He has average attachment with his father due to his authoritative nature as he was strict and demanding. His mother was a house wife. His parents had an arrange marriage but they were satisfied with their marital life. His mother parenting style was permissive as she demands low with high responsiveness. Moreover, she was very loving, and seemed more like a friend than a parental figure. So, due to his mother parenting style he had good attachment with his mother.

He was first born and lived in a nuclear family system in a rural area with his parents and 2 siblings (Brother; 1 , age; 15 years \& Sister; 1 , age; 17 years). He got education till intermediate (F.A). He was an average student throughout academic career. Before the time of diagnosis of CKD, he was driving his rickshaw. His brother was studying in $8^{\text {th }}$ class whereas his sister could not study. He seemed to be more attached to his sister as compared to his brother. His sister cared for him more than his brother.

He was living in village and his family was not so educated, due to these reasons they seemed to have family pattern of self-medication. According to the 
patients, he had constipation issue before the two months of the diagnosis CKD i.e. 2013. He did not seek treatment initially from any health service centers. When constipation problem did not resolve, and then he consults doctor. The doctor prescribed him medicines for constipation.

After passing two months with constipation without any recovery he consulted to another doctor from Lahore, who recommended him laboratory test i.e. creatanine and GFR. According to the medical reports he was diagnosed with CKD that was in reversible stage. Doctor told him that he would be cured if he took medicines properly. He used prescribed medicines for one month, the medicines did not cure him and creatinine level kept increasing i.e. $9 \mathrm{mg} / \mathrm{dl}$. He experiences loss of appetite, nausea or vomiting, itching and decrease volume of urine.

Creatnine and GFR ranges of the patient showed that he was at the fifth stage of CKD. He was given the option for renal transplantation but could not go for it due to the availability of living related kidney donor and financial constraints, as a result of which he got distressed. So he started Hemodialysis (HD) on thrice a week basis. Due to family pattern of self-medication late diagnosis of his conditions leads him towards dissatisfied doctor patient's relationship. This dissatisfied doctor patient relationship, family patterns of self-medications and economic status i.e. lower or poor leads him to non-adherence (medication, fluid and dietary).

$\mathrm{He}$ was seeking Hemodialysis services $150 \mathrm{~km}$ far from his home, due to un-availability of quality based Hemodialysis center in his city. The long travel and time constraints of the Hemodialysis regime resulted to be barrier for him for caring, as he could not continue his rickshaw driving as a result of which he had to face financial issues.

After detailed case history interview, he was psychologically assessed both formal and informal through behavioral observation along with illness perceptions, health focus of control, health beliefs, perceived health status, perception about health care services, and perception of doctor-patient relationship, life orientation, personality trait, psychological distress and adherence (medication, dietary and fluid) for psychological management. Target areas identified as result of psychosocial assessment included low socio-economic status, non-adherence (medication, fluid and dietary), dissatisfactory doctor patient relationship, pessimistic life orientation, and mal-adaptive health beliefs.

His management was based on psychodynamic, behavioral and cognitive approaches. His management plan was developed on health belief model. In the initial sessions rapport was tried to build up. The counseling was given to the patient by psycho-education regarding his dis-satisfactory doctor patient relationship. The counseling was given to the patient by maximizing the positive aspect of his life for his pessimistic life orientation. Coping statements was given to the patient to improve his self-efficacy.

The precipitating factor for health condition for his distress was his financial status i.e. low socioeconomic status and maintaining factor. This leads him towards distress. Successfully addressing the precipitating factors of his non-adherence, then he was psycho-educated around the cost and benefit analysis about effect of non-adherence on his fistula to deal his mal-adaptive health belief relevant to nonadherence. Furthermore, his mal-adaptive belief relevant to non-adherence was also dealt through $A B C$ model. The ABC Model is where - A stands for Antecedent (i.e. the situation that triggers the response) B stands for Beliefs (thoughts/interpretation of the situation/event) $\mathrm{C}$ stands for Consequences (the way we feel or behave). He improved himself relevant to his non-adherence by the end of $12^{\text {th }}$ session. In the last session family was psychoeducated for follow ups sessions. In follow-up sessions patient improved him towards the management of physical complaint including hypertension ranges, fatigue and shortening of breath.

\section{DISCUSSION}

In the present case, factors associated with good prognosis are: acceptance of the diagnosis and compliance on therapeutic interventions. Dissatisfactory doctor patient relationship, poor socioeconomic status and mal-adaptive health beliefs were contributing factors for poor prognoses.

Pervious literature suggests us those patients' beliefs about their disease and its treatment and quality of the doctor-patient relationship have been proposed to be central to adherence ${ }^{2}$. The patient demographic factor i.e. age and socio-economic status were also contributing factor for non-adherence. Previous studies demonstrated that demographic and socioeconomic factors including age (younger) and higher medication price leads patient towards nonadherence of doctor advice ${ }^{3}$. All these findings are consistent with the above-mentioned case.

Thus, strategies to improve adherence in CKD patient should primarily focus on the patient's trust developing by providing cost and benefits analysis and psychoeducate by giving as much information as possible on the necessity of taking all of these drugs ${ }^{5}$.

\section{CONCLUSION}

Under discussion patient was lack of poor communication with physicians, pessimistic life orientation, maladaptive health belief and cost of drugs seemed to be the reasons of non-adherence. This study found that $A B C$ model and cost/benefit analysis techniques to be effective for changing maladaptive health belief and improving his medication adherence, respectively. Psycho- 
education was helpful regarding his dissatisfied doctor patient relationship. Moreover, maximizing positive aspect of his life was effective for changing his pessimistic life orientation.

\section{REFERENCES}

1. Magacho EJ, Ribeiro LC, Chaoubah A, Bastos MG. Adherence to drug therapy in kidney disease. Braz J Med Biol Res. 2015; 44(3): 258-62. doi: 10.1590/s0100-879x2011007500013.

2. Greer DB, Ostwald S, Meininger JC, Taylor WC. Improving adherence in African American women with uncontrolled primary hypertension. Conference Paper. Conference: Scientific Sessions of High Blood Pressure Research. Hypertension. 2011; E120-E120.

3. Rasmussen JN, Chong A, Alter DA. Relationship between adherence to evidence-based pharmacotherapy and long-term mortality after acute myocardial infarction. JAMA. 2007; 297(2): 177-86. doi: 10.1001/jama.297.2.177.

4. Schneeweiss S, Patrick AR, Maclure M, Dormuth CR, Glynn RJ. Adherence to statin therapy under drug cost sharing in patients with and without acute myocardial infarction: a population-based natural experiment. Circulation. 2007; 115(16): 2128-35. doi: 10.1161/CIRCULATIONAHA.106.66 5992.

5. Rifkin DE, Laws MB, Rao M, Balakrishnan VS, Sarnak MJ, Wilson IB. Medication adherence behavior and priorities among older adults with CKD: a semi structured interview study. Am J Kidney Dis. 2010; 56(3): 439-46. doi:10.1053/ j.ajkd. 2010.04.021.

AUTHOR AFFILIATION:
Ghulam Ishaq (Corresponding Author)
Lecturer, Department of Psychology
Lahore Leads University-Lahore, Punjab-Pakistan.
Email: gishaq786@yahoo.com
Fatima Kamran
Assistant Professor
Institute of Applied Psychology
University of the Punjab, Lahore, Punjab-Pakistan.
Rafia Rafique
Associate Professor
Institute of Applied Psychology
University of the Punjab, Lahore, Punjab-Pakistan.
Shiza Sheerazi
Visiting Faculty Member,
Institute of Applied Psychology
University of the Punjab, Lahore, Punjab-Pakistan.

